\title{
BMJ Open Should HFE p.C282Y homozygotes with moderately elevated serum ferritin be treated? A randomised controlled trial comparing iron reduction with sham treatment (Mi-iron)
}

Sim Yee Ong, ${ }^{1,2}$ Lara Dolling, ${ }^{1}$ Jeannette L Dixon, ${ }^{3}$ Amanda J Nicoll, ${ }^{4,5}$ Lyle C Gurrin, ${ }^{6}$ Michelle Wolthuizen, ${ }^{1}$ Erica M Wood, ${ }^{7}$ Greg J Anderson, ${ }^{3}$ Grant A Ramm, ${ }^{8}$ Katrina J Allen, ${ }^{9,10}$ John K Olynyk, ${ }^{11}$ Darrell Crawford, ${ }^{12}$ Jennifer Kava, ${ }^{13}$ Louise E Ramm, ${ }^{8}$ Paul Gow, ${ }^{14}$ Simon Durrant, ${ }^{15}$ Lawrie W Powell, ${ }^{16}$ Martin B Delatycki ${ }^{1,17}$

To cite: Ong SY, Dolling L, Dixon JL, et al. Should HFE p.C282Y homozygotes with moderately elevated serum ferritin be treated? A randomised controlled trial comparing iron reduction with sham treatment (Miiron). BMJ Open 2015;5: e008938. doi:10.1136/ bmjopen-2015-008938

- Prepublication history for this paper is available online. To view these files please visit the journal online (http://dx.doi.org/10.1136/ bmjopen-2015-008938)

Received 3 June 2015 Accepted 17 July 2015

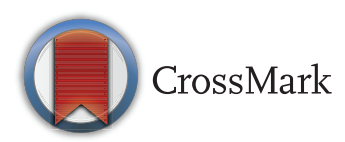

For numbered affiliations see end of article.

Correspondence to Professor Martin Delatycki; martin.delatycki@ghsv.org.au

\section{ABSTRACT}

Introduction: HFE p.C282Y homozygosity is the most common cause of hereditary haemochromatosis. There is currently insufficient evidence to assess whether non-specific symptoms or hepatic injury in homozygotes with moderately elevated iron defined as a serum ferritin (SF) of 300-1000 $\mu \mathrm{g} / \mathrm{L}$ are related to iron overload. As such the evidence for intervention in this group is lacking. We present here methods for a study that aims to evaluate whether non-specific symptoms and hepatic fibrosis markers improve with short-term normalisation of SF in p.C282Y homozygotes with moderate elevation of SF. Methods and analysis: Mi-iron is a prospective, multicentre, randomised patient-blinded trial conducted in three centres in Victoria and Queensland, Australia. Participants who are HFE p.C282Y homozygotes with SF levels between 300 and $1000 \mu \mathrm{g} / \mathrm{L}$ are recruited and randomised to either the treatment group or to the sham treatment group. Those in the treatment group have normalisation of SF by 3-weekly erythrocytapheresis while those in the sham treatment group have 3-weekly plasmapheresis and thus do not have normalisation of SF. Patients are blinded to all procedures. All outcome measures are administered prior to and following the course of treatment/sham treatment. Patient reported outcome measures are the Modified Fatigue Impact Scale (MFIS-primary outcome), Hospital Anxiety and Depression Scale (HADS), Medical Outcomes Study 36-item short form V.2 (SF36v2) and Arthritis Impact Measurement Scale 2 short form (AIMS2-SF). Liver injury and hepatic fibrosis are assessed with transient elastography (TE), Fibrometer and Hepascore, while oxidative stress is assessed by measurement of urine and serum F2isoprostanes.

Ethics and dissemination: This study has been approved by the Human Research Ethics Committees of Austin Health, Royal Melbourne Hospital and Royal
Brisbane and Women's Hospital. Study findings will be disseminated through peer-reviewed publications and conference presentations.

Trial registration: Trial identifier: NCT01631708; Registry: ClinicalTrials.gov

\section{INTRODUCTION}

Hereditary haemochromatosis $(\mathrm{HH})$, an iron overload disorder, is the most common genetic condition in Northern Europeans among whom approximately 1 in 200 are homozygous for the HFE p.C282Y amino acid substitution, the cause of most $\mathrm{HH}$. Not all p.C282Y homozygotes develop morbidity. ${ }^{1}$ About $20 \%$ of males and $40 \%$ of females with this genotype do not develop iron overload. $^{2}$ At least $28 \%$ of males and $1 \%$ of females develop iron overload-related disease such as liver cirrhosis, diabetes mellitus and cardiomyopathy. ${ }^{2}$ These individuals generally have severe iron overload as indicated by a serum ferritin (SF) of greater than $1000 \mu \mathrm{g} / \mathrm{L}$. Therefore, the question arises as to whether the largest group of p.C282Y homozygotes, those with raised SF but SF less than $1000 \mu \mathrm{g} / \mathrm{L}$ (defined here as moderate iron overload), require venesection treatment to normalise SF. Answering this question is important since if treatment is beneficial, introduction of community screening for $\mathrm{HH}$ should be considered whereas if there is no benefit from treatment, p.C282Y homozygotes with moderate iron overload do not need to undertake this somewhat onerous intervention. 
Major management guidelines for $\mathrm{HH}$ recommend treatment of those with $\mathrm{HH}$ and SF above the upper limit of normal and recommend that $\mathrm{SF}$ be reduced to 50 $100 \mu \mathrm{g} / \mathrm{L} .{ }^{3}{ }^{4}$ The rationale for normalisation of $\mathrm{SF}$ in those with severely elevated SF $(>1000 \mu \mathrm{g} / \mathrm{L})$ is clear as severe morbidity and mortality can be prevented. ${ }^{56}$ There is little evidence that treatment is beneficial in those with moderate iron overload however. There have been no randomised controlled trials to objectively assess whether returning iron levels to normal improves symptoms in $\mathrm{p}$. C282Y homozygotes with moderately elevated SF. Reasons why such studies have not been carried out likely include the fact that treatment of $\mathrm{HH}$ is relatively safe and therefore commentators have adopted the stance that treatment is unlikely to result in harm while there are theoretical reasons why harm may result from not normalising $\mathrm{SF}^{7} \mathrm{In}$ addition, blinding is far more complex in a trial of venesection than in a placebo-controlled pharmaceutical trial.

HFE p.C282Y homozygotes with moderately elevated SF do not have an increase in frequency of HH-related symptoms when compared to controls in cross-sectional studies. ${ }^{89}$ Such cross-sectional studies are not designed to identify subtle symptoms such as fatigue, however. Anecdotal evidence suggests that fatigue benefits from treatment of HH. Fatigue is a non-specific symptom that is commonly reported in individuals with $\mathrm{HH}^{2}{ }^{10-13}$ and has a negative impact on the physical and psychological quality of life. ${ }^{14}$ There are conflicting data about the relationship between fatigue and levels of excess iron. Adams et $a l^{10}$ found an association between fatigue and hepatic iron index in 410 p.C282Y homozygotes. In contrast, fatigue has been reported in p.C282Y homozygotes with normal $\mathrm{SF}^{1315}$ and has been found to be worse in some individuals following normalisation of $\mathrm{SF}$ by venesection treatment. ${ }^{11} 15$ One population study identified significantly higher Modified Fatigue Impact Scale (MFIS) scores in p.C282Y homozygotes who knew their diagnosis and had their iron levels normalised compared to those who were unaware of the diagnosis and had significantly higher $\mathrm{SF}^{2}{ }^{2}$ There are three possible explanations for these observations: (1) There is no relationship between fatigue and iron levels in HH; (2) there is a significant psychosomatic effect of diagnosis on how these individuals perceive fatigue; or (3) there is a subgroup of individuals who have fatigue and are more likely to be diagnosed with $\mathrm{HH}$.

A recent study suggested that treated HFE p.C282Y homozygotes with moderate iron overload have decreased cardiovascular and extrahepatic cancerrelated mortality rates compared to the general population, while p.C282Y homozygotes with normal SF have the same mortality rates as the general population. ${ }^{7}$ The relationship between the mortality findings and treatment of iron overload was questioned, however. ${ }^{16}$

\section{AIMS AND HYPOTHESIS}

The aim of this multicentre randomised trial is to compare the prevalence of symptoms and objective markers of disease between those in the treatment group and those in the sham treatment group.

Our hypothesis is that HFE p.C282Y homozygotes with moderately elevated SF will have few symptoms and signs of disease and decreasing SF to normal levels will not result in a greater change in patient reported outcomes or objective markers of liver injury or hepatic fibrosis compared to those whose SF levels are not lowered to the normal range.

\section{METHODS AND ANALYSIS}

The Mi-iron (Moderately increased iron levels) study is a multicentre, randomised single-blinded trial being conducted in Victoria (Austin Hospital and the Royal Melbourne Hospital) and Queensland (Royal Brisbane and Women's Hospital) that started in August 2012 and is due to conclude in December 2015. Figure 1 summarises the methodology of the Mi-iron Study.

\section{Participants}

Inclusion criteria

1. Age $18-70$ years inclusive

2. HFE p.C282Y homozygous

3. SF between 300 and $1000 \mu \mathrm{g} / \mathrm{L}$

4. Previously or currently raised TS

Exclusion criteria

1. HH due to other genotypes

2. Venesection in the past 2 years for treatment of $\mathrm{HH}$

3. Other risk factor(s) for liver injury including hepatitis B (HBV) or C (HCV), excess alcohol consumption $(>60 \mathrm{~g} /$ day in males, $40 \mathrm{~g} /$ day in females), body mass index (BMI) of $\geq 35 \mathrm{~kg} / \mathrm{m}^{2}$

4. Pregnant females

\section{Study intervention}

Apheresis is being used as the study intervention. All procedures are conducted using the Haemonetics MCS Plus apheresis system.

\section{Randomisation and stratification}

Participants are randomised to either the treatment group to have erythrocytapheresis or the sham treatment group to have plasmapheresis. Randomisation is by computer generated random number sequence. Randomisation is stratified by gender, initial SF $(300-599 \mu \mathrm{g} / \mathrm{L}$ or $600-1000 \mu \mathrm{g} / \mathrm{L}$ as a binary variable $)$ and site.

\section{Maintenance of blinding}

The participant is blinded as to which arm of the study he/she has been randomised by being connected to the apheresis machine with the machine and the tubing not visible to the individual. This is achieved by the participant's arm being passed through an opaque black curtain (figure 2). Thus participants are unaware of whether they are having red blood cells (RBCs) or plasma removed and are unaware of whether or not they 
Figure 1 Flow chart of methodology for the Mi-iron Study. TS, transferrin saturation; SF, serum ferritin; MFIS, Modified Fatigue Impact Scale; SF36v2, Medical Outcomes Study 36-item short form V.2; HADS, Hospital Anxiety and Depression scale; Measurement Scales 2 short form; TE, transient elastography. AIMS2-SF, Arthritis Impact

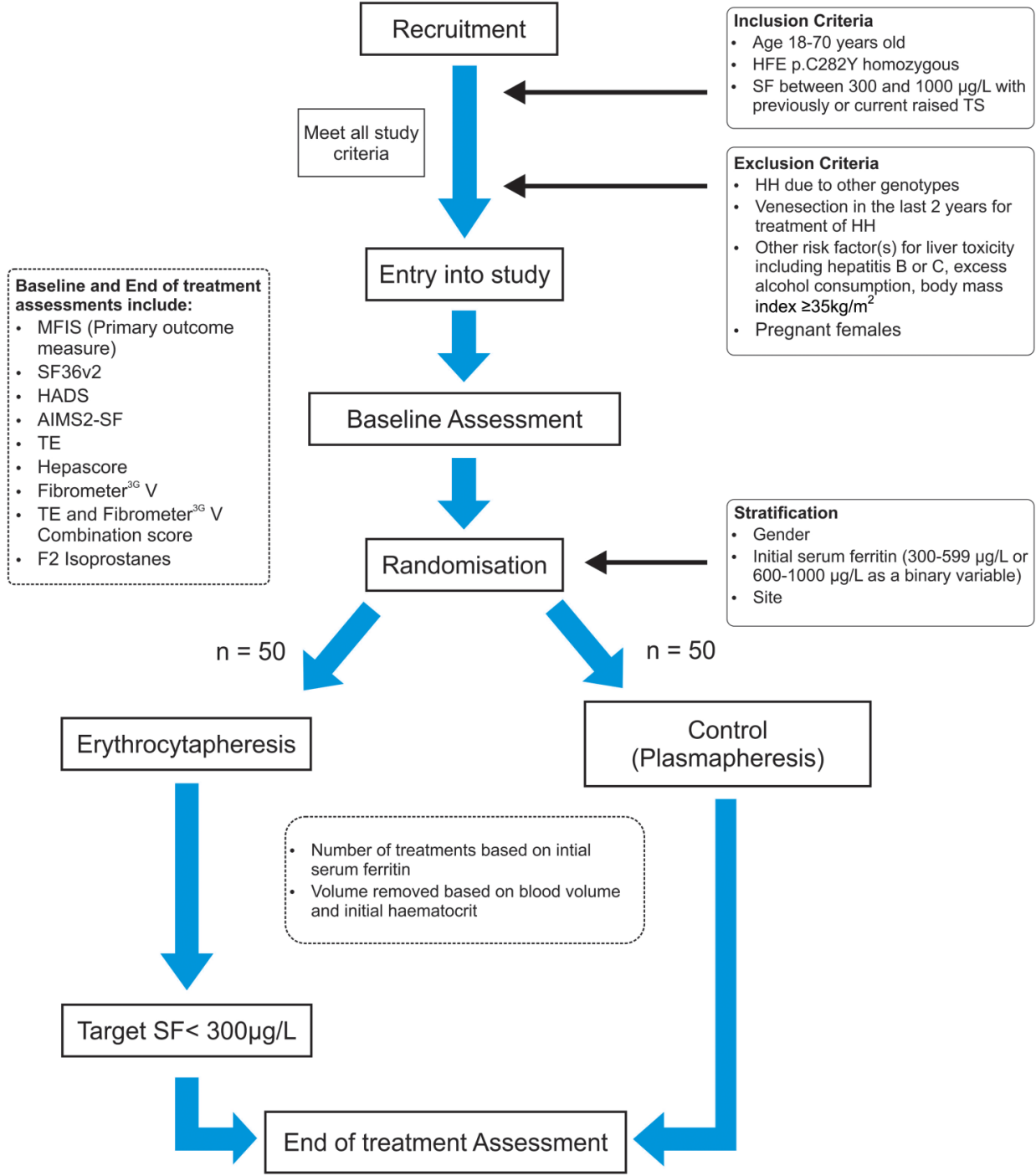

are having their iron levels reduced. Staff performing the apheresis are trained to not inadvertently reveal the treatment arm of the participant through strict adherence to study protocol and careful use of language in describing what is being performed. A member of the research team is present during the intervention to ensure blinding is maintained by monitoring the procedure and conversation between apheresis staff with the participant.

Those undergoing plasmapheresis have the same volume of plasma removed as the volume of RBCs removed had they been randomised to the erythrocytapheresis arm. Therefore, the risk of symptoms due to reduction in circulating blood volume is the same for individuals in both arms of the study.

\section{Intervention}

Treatments are administered every 3 weeks. The volume of RBCs/plasma removed is individualised based on the individual's blood volume and haematocrit. Haematocrit is measured at the start of each erythrocytapheresis treatment while in the sham treatment group, mock blood tests are taken to ensure the participant's experience is identical irrespective of the treatment group to which they have been randomised. The volume removed is calculated based on the height, weight, pretreatment haematocrit and the target haematocrit (30-35\%; figure $3)$. Treatments are ceased in the treatment group when $\mathrm{SF}$ is below $300 \mu \mathrm{g} / \mathrm{L}$. For the sham group, the calculated number of treatments is equivalent to as if their SF was normalised in the RBC group.

Participants in the sham arm are offered erythrocytapheresis or phlebotomy to normalise SF at their choice of venue on completion of the study.

\section{Safety blood test monitoring}

All participants have SF measured 2-3 weeks after the expected last treatment to ensure the SF level has decreased to the normal range $(<300 \mu \mathrm{g} / \mathrm{L})$ for those in the treatment group before proceeding to the end of trial assessment. Participants in both arms of the study have the same blood test to ensure blinding. Serum B12, 

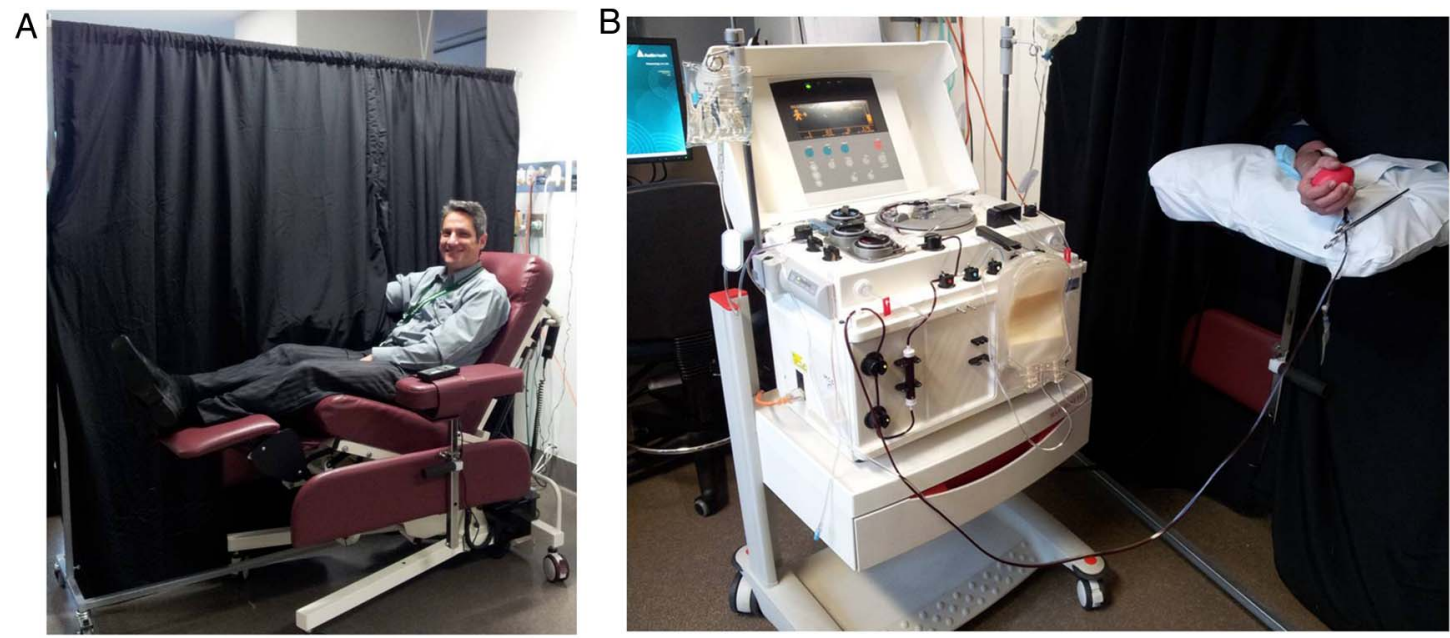

Figure 2 A black opaque curtain prevents the participant from seeing the apheresis machine and therefore the individual cannot see if red blood cells or plasma is removed. (A) View from the patient's perspective, (B) View from the apheresis machine side of the curtain.

folate, iron studies and full-blood count are checked approximately mid-treatment in both groups for safety monitoring.

\section{Target SF}

There have been no definitive studies conducted to demonstrate what the target SF should be at the end of treatment in an individual with $\mathrm{HH}$. Guidelines recommend a target SF of less than 50-100 $\mu \mathrm{g} / \mathrm{L}$. In this study, we have chosen to reduce SF to anywhere in the normal range, that is, a SF of $20-300 \mu \mathrm{g} / \mathrm{L}$, based on the expectation that an individual with $\mathrm{HH}$ whose $\mathrm{SF}$ is in the normal range should have similar symptoms to those without $\mathrm{HH}$ and who have a normal SF.

\section{Outcome measures}

The outcome measures being assessed are patientreported outcome scales to assess symptoms, as well as markers of liver injury, hepatic fibrosis and oxidative stress. These are administered at baseline and the end of erythrocytapheresis/ sham apheresis treatment.

\section{Patient-reported outcome scales}

1. Modified Fatigue Impact Scale (MFIS) is the primary outcome measure for this study. The MFIS is a 21-item scale that measures the impact of fatigue on three independent subscales of physical, cognitive and psychosocial functioning. ${ }^{18}$ Participants rate their fatigue in the past month on a five-point Likert-type scale. The total score ranges from 0 to 84 with higher scores reflecting greater fatigue. Subscale scores, physical (0-36); cognitive (0-40); and psychosocial $(0-8)$, can also be derived.

2. Medical Outcomes Study 36-item short form V.2 (SF36v2) is a 36-item generic health survey to measure health and well-being ${ }^{19}$ that has been previously used in various $\mathrm{HH}$ studies to measure quality of life. ${ }^{2} 122021$ It assesses eight different health components (physical functioning, role-physical, bodily pain, general health, vitality, social functioning, role-emotional, mental health) and provides a summary score for both physical and mental components. It is a normbased scoring system and thus can be used to compare participant scores to the general population.

3. Hospital Anxiety and Depression Scale (HADS) is composed of a 14-item total scale (HADS-T) consisting of two seven-item independent subscales, the Anxiety (HADS-A) and Depression (HADS-D) subscales. ${ }^{22}$ Participants rate how they have felt in the past week on a four-point Likert-type scale. Scores on each scale can be interpreted in ranges: normal $(0-7)$; mild (8$10)$; moderate (11-14); and severe (15-21). Higher scores on each subscale or the entire scale indicate
Figure 3 Equation to estimate postcollection haematocrit (Hct post) based on total blood volume (TBV) using Nadler's fomula. ${ }^{17}$ Adopted from the Haemonetics MCS Plus apheresis system manual. TBV, total blood volume; RBC, red blood cell; Hct, haematocrit.

\section{Hct post $=$ \\ (TBV $x$ Donor Hct/100) - (Target RBC volume $\times$ Bowl Hct/100) TBV - Target RBC volume + Compensation Volume $+(30 \mathrm{ml} x$ cycles $)$}

Nadler's Formula

Female TBV $(\mathrm{ml})=183+0.000356 \times$ height $(\mathrm{cm})^{3}+33 \times$ weight $(\mathrm{kg})$

Male TBV $(\mathrm{ml})=604+0.0003668 \times$ height $(\mathrm{cm})^{3}+32.2 \times$ weight $(\mathrm{kg})$ 
greater anxiety, depression or both. This scale has been found to be valid and reliable in various populations. $^{2324}$

4. Arthritis Impact Measurement Scales 2 short form (AIMS2-SF) is a 26-item validated scale that assesses the impact of arthritis on five core domains of the participants. ${ }^{25} 26$ It measures physical functioning, symptoms, affect, role and social interactions of the individuals. A five-point Likert-type scale is used to rate how participants have felt in the past month. The higher the raw score, the greater the impact of arthritis on the participant. Use of arthritis medication at baseline and end of trial will also be compared.

5. To assess the fidelity of the blinding process, the participants are asked which treatment group they believe they were allocated to at the completion of the study, before unblinding.

\section{Liver injury and hepatic fibrosis markers}

Transient elastography (TE) and blood tests for components of Hepascore and Fibrometer ${ }^{3 \mathrm{G}} \mathrm{V}$ are collected from individuals at baseline and end of the trial.

\section{Transient elastography}

Fibroscan is a method of TE that evaluates liver stiffness using an ultrasound probe to measure the velocity of a mechanical wave that is pulsed through the liver. As the liver becomes progressively more fibrotic, it becomes harder and less elastic. The velocity of the wave correlates directly with tissue stiffness and results are reported in kilopascals $(\mathrm{kPa}) .{ }^{27} \mathrm{TE}$ has been evaluated in a number of different liver diseases, including HBV and $\mathrm{HCV}$, alcoholic liver disease, non-alcoholic fatty liver disease and $\mathrm{HH}^{28}{ }^{29} \mathrm{~A}$ recent meta-analysis of nine studies involving TE showed excellent results for diagnosing cirrhosis, with a sensitivity of $87 \%$ and specificity of $91 \% .^{30}$ Adhoute $e t a l^{31}$ have shown that TE measurements correlate with Hepascore measurements in individuals with HH. The results of liver stiffness are acquired from at least 10 successful valid measurements with a success rate of at least $60 \%$ within the IQR of $\leq 30 \%$. A cut-off value of $8.7 \mathrm{kPa}$ was sensitive for the diagnosis of those with significant fibrosis $(\geq F 2)$, with an area under the curve (AUC) for the receiver operating characteristic (ROC) of $0.79^{32}$ and a reading of more than $13 \mathrm{kPa}$ was highly predictive that cirrhosis of the liver was present in a cohort with iron overload due to $\beta$-thalassaemia. ${ }^{33}$

\section{Hepascore}

Hepascore is derived from an age-specific and genderspecific model that inputs parameters of serum bilirubin, $\gamma$ glutamyl transferase (GGT), hyaluronic acid and $\alpha 2$-macroglobulin. The test results in a score between 0 and 1 with a higher score being associated with more severe liver disease. In a HCV cohort, Hepascore demonstrated an AUC for the ROC of 0.8 for predicting significant fibrosis $(\geq \mathrm{F} 2)$ and 0.90 for predicting cirrhosis. ${ }^{34}$ A score $>0.5$ was found to have a specificity of $70 \%$ and sensitivity of $77 \%$ to detect significant fibrosis $(\geq \mathrm{F} 2)$ in a large HCV cohort. ${ }^{35}$ A Hepascore $<0.25$ can exclude significant fibrosis with a negative predictive value of $0.9 .^{35}$ In a study that included the Hepascore in HFE related HH, 44 p.C282Y homozygotes had a median score of $0.1 .^{36}$

\section{Fibrometer $^{3 \mathrm{G}} \mathrm{V}$}

Fibrometer $^{3 \mathrm{G}} \mathrm{V}$ is formulated from the platelet count (PLT), prothrombin index (PI), and the alanine amino transaminase (ALT), aspartate amino transaminase (AST), GGT, $\alpha 2-$ macroglobulin and urea levels. This biomarker had an AUROC of 0.85 for predicting significant fibrosis and an AUROC of 0.9 for predicting cirrhosis in a HCV cohort. ${ }^{37}$ Its robustness has been evaluated in different studies and has been recommended by the French National Authority for Health for the estimation of liver fibrosis in HCV.

The combination of Fibrometer ${ }^{3 \mathrm{G}} \mathrm{V}$ and $\mathrm{TE}$ has recently been shown to increase the accuracy of diagnosing significant fibrosis and cirrhosis to $92 \%$ compared with Fibrometer ${ }^{3 \mathrm{G}} \mathrm{V}(84 \%$ accuracy) or TE $(88 \%$ accuracy) alone. The combination has an AUROC of 0.89, improving the reliability and precision of diagnosis of significant fibrosis in chronic liver disease. ${ }^{38} 39$ Fibrometer has not been tested in HH.

\section{Oxidative stress marker}

Iron is a strong pro-oxidant and there is evidence that markers of oxidative stress are elevated in individuals with elevated iron indices due to $\mathrm{HH}^{40-43}$ To assess oxidative stress, F2-isoprostanes, a validated marker of cellular lipid oxidative damage, ${ }^{44}$ are being measured in urine and blood. While elevated makers of oxidative stress are not necessarily related to symptoms of disease, we will be able to assess the relationship between this early marker of tissue injury and the other markers being measured, including iron indices, Hepascore, Fibrometer, TE score and the scores for the various clinical scales being administered. We will also assess whether F2-isoprostanes are positively impacted by normalisation of iron indices in the erythrocytapheresis group.

\section{Sample size calculation}

Data from the Melbourne HealthIron study ${ }^{2}$ were used to calculate SDs of the MFIS score of 14.1 and 17.8 for male and female C282Y homozygotes, respectively. Using a conservative value for the SD of 18 , a sample size of 50 in each treatment group ensures an $80 \%$ chance (statistical power) that a treatment effect of a mean difference of 10 MFIS units (well above a clinically relevant difference on this scale which runs from 0 to 84 ) is reflected in a $\mathrm{p}$ value less than 0.05 . Summary statistics from figure 2 of Adams $e t a l^{34}$ show the mean Hepascore changing from 0.20 in patients with METAVIR fibrosis grade 0 or 1 (F0 or F1), through 0.45 in those with F2, to close to 1.0 in 
those with F3 or F4. The within-fibrosis grade SD of Hepascore is approximately 0.20 in the F2 group and it is much lower in the remaining groups. Using this SD, a sample size of 50 in each treatment group of the trial delivers statistical power of $85 \%$ to detect a treatment effect of 0.12 on the Hepascore scale. A change of this magnitude is similar to the observed mean difference in Hepascore between adjacent fibrosis groups presented in Adams $e t a l .{ }^{34}$ Accommodating the stratified design, the regression-based statistical analysis will result in minimal loss of power provided that only the average initial measures and not the treatment effects are different between strata.

\section{Statistical analysis}

The primary analysis addressing the research hypothesis will be a comparison of the change in scores for all outcome scales, biochemical tests and TE scores, from baseline to end of treatment, between those who have had their iron levels returned to normal and those who were in the sham treatment group, with assignment to the comparison groups based on intention to treat. This analysis will be implemented using a linear regression model of the final measure on each scale including as covariates the value of the initial measure, gender, initial $\mathrm{SF}(300-599 \mu \mathrm{g} / \mathrm{L}$ or $600-1000 \mu \mathrm{g} / \mathrm{L}$ as a binary variable) and site (Melbourne or Brisbane as a two category variable). In a separate analysis, this model with the Hepascore as the outcome measure will be extended to include the quantity of iron removed (calculated as $1 \mathrm{~g}$ iron per litre of RBCs removed by erythrocytapheresis) to determine whether there is an association between the change in Hepascore and the reduction in iron level. Similar analyses will be performed for F2-isoprostanes, TE and patient-reported outcome scales.

\section{CONCLUSION}

This is the first randomised controlled trial of treatment for $\mathrm{HH}$. It will demonstrate whether there is any benefit in the short term from normalisation of SF in HFE $p$. C282Y homozygotes with moderately elevated SF. This has implications for management of this group of individuals and may assist in determining whether introduction of community screening for $\mathrm{HH}$ should be considered.

\footnotetext{
Author affiliations

${ }^{1}$ Bruce Lefroy Centre, Murdoch Childrens Research Institute, Parkville, Victoria, Australia

${ }^{2}$ Department of Medicine, The University of Melbourne, Parkville, Victoria, Australia

${ }^{3}$ Iron Metabolism Group, QIMR Berghofer Medical Research Institute, Herston, Queensland, Australia

${ }^{4}$ Department of Gastroenterology, Eastern Health, Box Hill, Victoria, Australia

${ }^{5}$ Department of Gastroenterology, Royal Melbourne Hospital, Parkville, Victoria, Australia

${ }^{6}$ Centre for Epidemiology and Biostatistics, Melbourne School of Population and Global Health, The University of Melbourne, Parkville, Victoria, Australia ${ }^{7}$ Transfusion Research Unit, Department of Epidemiology and Preventive Medicine, Monash University, Prahran, Victoria, Australia
}

${ }^{8}$ Hepatic Fibrosis Group, QIMR Berghofer Medical Research Institute, Herston, Queensland, Australia

${ }^{9}$ Gastro and Food Allergy, Murdoch Childrens Research Institute, Parkville,

Victoria, Australia

${ }^{10}$ Allergy and Immunology, Royal Children's Hospital, Parkville, Victoria, Australia

${ }^{11}$ Department of Gastroenterology, Fiona Stanley and Fremantle Hospitals, Murdoch, Western Australia, Australia

${ }^{12}$ School of Medicine, University of Queensland, Herston, Queensland, Australia

${ }^{13}$ Department of Gastroenterology, Fremantle Hospital, Alma St, Fremantle, Western Australia, Australia

${ }^{14}$ Department of Gastroenterology, Austin Health, Heidelberg, Victoria, Australia

${ }^{15}$ Bone Marrow Transplant and Haematology, Royal Brisbane Hospital, Herston, Queensland, Australia

${ }^{16}$ RBWH Centre for the Advancement of Clinical Research, Royal Brisbane \& Women's Hospital, Herston, Queensland, Australia

${ }^{17}$ Clinical Genetics, Austin Health, Heidelberg, Victoria, Australia

Twitter Follow Jeannette Dixon at @Jade_45

Contributors SYO, LD, LCG and MBD wrote the first draft of the manuscript. SYO is involved in participant recruitment and assessment. LD and MW coordinate the study in Melbourne and are responsible for data management. JLD and LER coordinate the study in Brisbane. AJN and LWP participated in design of the trial and are involved in participant recruitment and assessment. LCG contributed to trial design and is the study statistician involved in randomisation and data analysis. EMW, GJA, GAR, KJA, JKO, DC, JK, PG and $S D$ contributed to design of the trial. MBD is the chief investigator of the study, conceived the study, was involved in design of the study and is involved in recruitment and assessment of participants.

Funding This project is funded by the National Health and Medical Research Council of Australia, grant number 1026394. We acknowledge the contribution of Haemonetics Australia for provision of apheresis machines for the study and Echosens who will provide Fibrometer calculations free of charge. This work was assisted by Victorian State Government Operational Infrastructure Support and Australian Government NHMRC IRIISS.

Competing interests None declared.

Ethics approval This study has been approved by the Human Research Ethics Committees of Austin Health, Royal Melbourne Hospital and Royal Brisbane and Women's Hospital.

Provenance and peer review Not commissioned; peer reviewed for ethical and funding approval prior to submission.

Open Access This is an Open Access article distributed in accordance with the Creative Commons Attribution Non Commercial (CC BY-NC 4.0) license, which permits others to distribute, remix, adapt, build upon this work noncommercially, and license their derivative works on different terms, provided the original work is properly cited and the use is non-commercial. See: http:// creativecommons.org/licenses/by-nc/4.0/

\section{REFERENCES}

1. Feder JN, Gnirke A, Thomas W, et al. A novel MHC class I-like gene is mutated in patients with hereditary haemochromatosis. Nat Genet 1996;13:399-408

2. Allen KJ, Gurrin LC, Constantine CC, et al. Iron-overload-related disease in HFE hereditary hemochromatosis. $N$ Engl J Med 2008;358:221-30.

3. Bacon BR, Adams PC, Kowdley KV et al. American Association for the Study of Liver D. Diagnosis and management of hemochromatosis: 2011 practice guideline by the American Association for the Study of Liver Diseases. Hepatology 2011;54:328-43.

4. EASL. EASL clinical practice guidelines for HFE hemochromatosis. $J$ Hepatol 2010;53:3-22.

5. Adams PC, Speechley M, Kertesz AE. Long-term survival analysis in hereditary hemochromatosis. Gastroeneterology 1991;101:368-72. 
6. Niederau C, Fischer R, Purschel A, et al. Long-term survival in patients with hereditary hemochromatosis. Gastroenterology 1996;110:1107-19.

7. Bardou-Jacquet E, Morcet J, Manet G, et al. Decreased cardiovascular and extrahepatic cancer-related mortality in treated patients with mild HFE hemochromatosis. J Hepatol 2015;62:682-9.

8. Allen KJ, Bertalli NA, Osborne NJ, et al. HFE Cys282Tyr homozygotes with serum ferritin concentrations below 1000 microg/L are at low risk of hemochromatosis. Hepatology 2010;52: 925-33.

9. Andersen RV, Tybjaerg-Hansen A, Appleyard M, et al. Hemochromatosis mutations in the general population: iron overload progression rate. Blood 2004;103:2914-19.

10. Adams PC, Deugnier Y, Moirand R, et al. The relationship between iron overload, clinical symptoms, and age in 410 patients with genetic hemochromatosis. Hepatology 1997;25:162-6.

11. McDonnell SM, Preston BL, Jewell SA, et al. A survey of 2,851 patients with hemochromatosis: symptoms and response to treatment. Am J Med 1999;106:619-24.

12. Delatycki MB, Allen KJ, Nisselle AE, et al. Use of community genetic screening to prevent HFE-associated hereditary haemochromatosis. Lancet 2005;366:314-16.

13. McLaren GD, McLaren CE, Adams PC, et al. Clinical manifestations of hemochromatosis in HFE C282Y homozygotes identified by screening. Can J Gastroenterol 2008;22:923-30.

14. Lowry TJ, Pakenham KI. Health-related quality of life in chronic fatigue syndrome: predictors of physical functioning and psychological distress. Psychol Health Med 2008;13:222-38.

15. Niewiadomski O, Rode A, Bertalli N, et al. The effectiveness of venesection therapy for haemochromatosis symptoms. J Liver Dis Transplant 2013;2:1-5.

16. Delatycki MB, Gurrin LC, Ong SY, et al. Reduced mortality due to phlebotomy in moderately iron-loaded HFE Haemochromatosis? The need for clinical trials. J Hepatol 2015;63:282-3.

17. Nadler SB, Hidalgo JH, Bloch T. Prediction of blood volume in normal human adults. Surgery 1962;51:224-32.

18. Fisk JD, Ritvo PG, Ross L, et al. Measuring the functional impact of fatigue: initial validation of the fatigue impact scale. Clin Infect Dis 1994;18(Suppl 1):S79-83.

19. Ware JE, Jr., Sherbourne CD. The MOS 36-item short-form health survey (SF-36). I. Conceptual framework and item selection. Med Care 1992;30:473-83.

20. Meiser B, Dunn S, Dixon J, et al. Psychological adjustment and knowledge about hereditary hemochromatosis in a clinic-based sample: a prospective study. J Genet Couns 2005;14:453-63.

21. Power TE, Adams PC, Barton JC, et al. Psychosocial impact of genetic testing for hemochromatosis in the HEIRS Study: a comparison of participants recruited in Canada and in the United States. Genet Test 2007;11:55-64.

22. Zigmond AS, Snaith RP. The hospital anxiety and depression scale. Acta Psychiatr Scand 1983;67:361-70.

23. Bjelland I, Dahl AA, Haug TT, et al. The validity of the Hospital Anxiety and Depression Scale. An updated literature review. J Psychosom Res 2002;52:69-77.

24. McDowell I. Measuring health: a guide to rating scales and questionnaires. 3rd edn. New York: Oxford University Press, 2006.

25. Ren XS, Kazis L, Meenan RF. Short-form Arthritis Impact Measurement Scales 2: tests of reliability and validity among patients with osteoarthritis. Arthritis Care Res 1999;12:163-71.
26. ten Klooster PM, Veehof MM, Taal E, et al. Confirmatory factor analysis of the Arthritis Impact Measurement Scales 2 short form in patients with rheumatoid arthritis. Arthritis Rheum 2008;59:692-8.

27. Andersen ES, Christensen PB, Weis N. Transient elastography for liver fibrosis diagnosis. Eur J Intern Med 2009;20:339-42.

28. Friedrich-Rust M, Ong MF, Martens S, et al. Performance of transient elastography for the staging of liver fibrosis: a meta-analysis. Gastroenterology 2008;134:960-74.

29. Manning DS, Afdhal NH. Diagnosis and quantitation of fibrosis. Gastroenterology 2008;134:1670-81.

30. Talwalkar JA, Kurtz DM, Schoenleber SJ, et al. Ultrasound-based transient elastography for the detection of hepatic fibrosis: systematic review and meta-analysis. Clin Gastroenterol Hepatol 2007;5:1214-20.

31. Adhoute X, Foucher J, Laharie D, et al. Diagnosis of liver fibrosis using FibroScan and other noninvasive methods in patients with hemochromatosis: a prospective study. Gastroenterol Clin Biol 2008;32:180-7.

32. Ziol M, Handra-Luca A, Kettaneh A, et al. Noninvasive assessment of liver fibrosis by measurement of stiffness in patients with chronic hepatitis C. Hepatology 2005;41:48-54.

33. Di Marco V, Bronte F, Cabibi D, et al. Noninvasive assessment of liver fibrosis in thalassaemia major patients by transient elastography (TE)-lack of interference by iron deposition. $\mathrm{Br} J$ Haematol 2010;148:476-9.

34. Adams LA, Bulsara M, Rossi E, et al. Hepascore: an accurate validated predictor of liver fibrosis in chronic hepatitis $C$ infection. Clin Chem 2005;51:1867-73.

35. Guechot J, Lasnier E, Sturm N, et al. group AHEFs. Automation of the Hepascore and validation as a biochemical index of liver fibrosis in patients with chronic hepatitis $C$ from the ANRS HC EP 23 Fibrostar cohort. Clin Chem Acta 2010;411:86-91.

36. Pankow JS, Boerwinkle E, Adams PC, et al. HFE C282Y homozygotes have reduced low-density lipoprotein cholesterol: the Atherosclerosis Risk in Communities (ARIC) Study. Transl Res 2008;152:3-10.

37. Cales P, Boursier J, Bertrais S, et al. Optimization and robustness of blood tests for liver fibrosis and cirrhosis. Clin Biochem 2010;43:1315-22

38. Boursier J, Vergniol J, Sawadogo A, et al. The combination of a blood test and Fibroscan improves the non-invasive diagnosis of liver fibrosis. Liver Int 2009;29:1507-15.

39. Cales $P$, Boursier J, Ducancelle A, et al. Improved fibrosis staging by elastometry and blood test in chronic hepatitis C. Liver Int 2014;34:907-17.

40. Broedbaek K, Poulsen HE, Weimann A, et al. Urinary excretion of biomarkers of oxidatively damaged DNA and RNA in hereditary hemochromatosis. Free Radic Biol Med 2009;47:1230-3.

41. Houglum K, Ramm GA, Crawford DH, et al. Excess iron induces hepatic oxidative stress and transforming growth factor beta1 in genetic hemochromatosis. Hepatology 1997;26:605-10.

42. Kom GD, Schwedhelm E, Nielsen P, et al. Increased urinary excretion of 8-iso-prostaglandin F2alpha in patients with HFE-related hemochromatosis: a case-control study. Free Radic Biol Med 2006;40:1194-200.

43. Shizukuda Y, Bolan CD, Nguyen TT, et al. Oxidative stress in asymptomatic subjects with hereditary hemochromatosis. $A m ~ J$ Hematol 2007;82:249-50.

44. Basu S. F2-isoprostanes in human health and diseases: from molecular mechanisms to clinical implications. Antioxid Redox Signal 2008;10:1405-34. 